\title{
Remarks on the Conditional Quadratic and the Conditional D'Alembert Functional Equations on Particular Normed Spaces
}

\author{
Margherita Fochi and Gabriella Viola \\ Dipartimento di Matematica, Università di Torino, Via Carlo Alberto 10, I-10123 Torino, Italy \\ Correspondence should be addressed to Margherita Fochi; margherita.fochi@unito.it
}

Received 20 March 2013; Accepted 6 June 2013

Academic Editor: Pedro M. Lima

Copyright (C) 2013 M. Fochi and G. Viola. This is an open access article distributed under the Creative Commons Attribution License, which permits unrestricted use, distribution, and reproduction in any medium, provided the original work is properly cited.

Let $X$ be a real normed space with dimension greater than 2 and let $f$ be a real functional defined on $X$. Applying some ideas from the studies made on the conditional Cauchy functional equation on the restricted domain of the vectors of equal norm and the isosceles orthogonal vectors, the conditional quadratic equation and the D'Alembert one, namely, $\|x\|=\|y\| \Rightarrow f(x+y)+f(x-y)=$ $2 f(x)+2 f(y)$ and $\|x\|=\|y\| \Rightarrow f(x+y)+f(x-y)=2 f(x) f(y)$, have been studied in this paper, in order to describe their solutions. Particular normed spaces are introduced for this aim.

\section{Introduction}

The conditional functional equation

$$
\|x\|=\|y\| \Longrightarrow f(x+y)=f(x)+f(y),
$$

where $f: X \rightarrow Y$ is a continuous mapping from an inner product space $X$ of dimension $\geq 2$ into a real topological vector space $Y$, was first studied by Alsina and Garcia Roig in [1]. They recognized the connection between this equation and the orthogonally additive functional equation that is

$$
x \perp y \Longrightarrow f(x+y)=f(x)+f(y)
$$

which is a conditional functional equation studied by several authors and for long time (see among others [2-5]). In particular we mention the general results obtained by Rätz [3]: he described the solutions of (ii) in the so-called orthogonality spaces in which the orthogonality relation is defined in axiomatic way and it is homogeneous.

Then Szabó [6] studied the previous (i) for functionals $f:(X,\|\cdot\|) \rightarrow(Y,+)$ defined in a real normed space with values in an abelian group. In this paper he considered also the following conditional equation:

$$
x \perp_{i} y \Longrightarrow f(x+y)=f(x)+f(y)
$$

which is the additive Cauchy functional equation on the restricted domain of the isosceles orthogonal vectors in the sense of James [7]; that is, $x, y$ are isosceles orthogonal $x \perp_{i} y$ if $\|x+y\|=\|x-y\|$.

This study of functional equations on the restricted domain of the vectors of equal norm or the isosceles orthogonal vectors in linear normed spaces, which are not inner product spaces, is quite difficult.

In particular, the lack of homogeneity for the isosceles orthogonality leads to the search for a new different approach for the proof, since the general theory of the orthogonality spaces in the sense of Rätz does not cover this case. The method of the proof of those theorems is by no means elementary. In particular, some sophisticated connectivity results on intersection of spheres of equal radii in normed linear spaces are involved. The proofs are based on the existence of particular auxiliary vectors of equal norm in normed spaces. Moreover, the method spoken of requires the dimension of the space considered to be greater than or equal to 3 .

The aim of our paper is to consider two other functional equations: the quadratic functional equation

$$
(x, y) \in X^{2} \Longrightarrow f(x+y)+f(x-y)=2 f(x)+2 f(y)
$$

and the D'Alembert functional equation

$$
(x, y) \in X^{2} \Longrightarrow f(x+y)+f(x-y)=2 f(x) f(y)
$$


and to study the solutions of their conditional version on the restricted domain of vectors of equal norm in the class of functionals defined on a normed space; this is the starting point. In the future we purpose to study the above conditional equations on the restricted domain of the isosceles orthogonal vectors.

Let $f:(X,\|\cdot\|) \rightarrow R$. We study the following conditional equations:

$$
\begin{gathered}
\|x\|=\|y\| \Longrightarrow f(x+y)+f(x-y)=2 f(x)+2 f(y), \\
\|x\|=\|y\| \Longrightarrow f(x+y)+f(x-y)=2 f(x) f(y)
\end{gathered}
$$

taking hint from the techniques introduced in the previous papers, based on suitable vectors in normed spaces.

We notice that the additive equation involves the values of $f$ on the vectors $x, y, x+y$, and the auxiliary vectors introduced by Szabó can perform in this case. For (3) and (4) we have to deal with the values of $f$ on $x, y$ and both vectors $x+y$ and $x-y$. So we should assume the existence of useful vectors, defining particular normed spaces in which such vectors exist. We describe those spaces in Section 2.

Afterwards, in Sections 3 and 4, we study the conditional quadratic equation and the D'Alembert equation, respectively, in those spaces, obtaining a characterization of its solutions.

Then, in Section 5, we consider the even isosceles orthogonal additive functionals in the particular class of the functional defined on the normed spaces introduced in Section 2, and we give their representation in order to characterize the particular normed spaces introduced in this paper; unfortunately this characterization remains since now an open problem.

\section{Particular Normed Spaces}

Let $(X,\|\cdot\|)$ be a normed space $X$ with $\operatorname{dim} X \geq 3$. Following the ideas of Szabó, let us introduce in $X$ some auxiliary vectors by the following conditions.

Let $x, y \in X$ be vectors with $\|x\|<\|y\|$; then there exist $z_{1}, z_{2} \in X$ such that

$$
\begin{gathered}
2 x=z_{1}+z_{2}, \\
\|y\|=\left\|z_{1}\right\|=\left\|z_{2}\right\|, \\
\left\|y+z_{1}\right\|=\left\|y+z_{2}\right\| .
\end{gathered}
$$

Let $x, y \in X$ be vectors with $\|x\|<\|y\|$; then there exist $z_{3}, z_{4} \in X$ such that

$$
\begin{gathered}
2 x=z_{3}+z_{4}, \\
\|y\|=\left\|z_{3}\right\|=\left\|z_{4}\right\|, \\
\left\|y-z_{3}\right\|=\left\|y-z_{4}\right\| .
\end{gathered}
$$

Let $x, y \in X$ be vectors with $\|x\|<\|y\|$; then there exist $x_{1}, x_{2} \in X$ such that

$$
\begin{gathered}
2 x=x_{1}+x_{2}, \\
\|y\|=\left\|x_{1}\right\|=\left\|x_{2}\right\|, \\
\left\|y+x_{1}\right\|=\left\|y+x_{2}\right\|, \\
\left\|y-x_{1}\right\|=\left\|y-x_{2}\right\| .
\end{gathered}
$$

We can also use the following condition $\left(C^{\prime}\right)$ which is a condition equivalent to $(\mathrm{C})$.

Let $x, y \in X$ be vectors with $\|x\|<\|y\|$; then there exists $z \in X$ such that

$$
\begin{gathered}
\|y\|=\|z\|=\|2 x-z\|, \\
\|y+z\|=\|y+(2 x-z)\|, \\
\|y-z\|=\|y-(2 x-z)\| .
\end{gathered}
$$

In fact, it is sufficient to consider in (C) $x_{1}=z$; hence $x_{2}=$ $2 x-z$.

Condition (A) has been introduced by Szabó [6] and the existence of such vectors is proved in every normed spaces, as we can see from the following.

Proposition 1. Let $(X,\|\cdot\|)$ be a normed space $X$ with $\operatorname{dim} X \geq$ 3 , then condition (A) is satisfied.

Proof. Let $x, y \in X$ be vectors with $\|x\|<\|y\|$. We put $\|y\|=$ $\rho$; consider $K$ the connected intersection of spheres $S_{0}(\rho)=$ $\{z \in X /\|x\|=\rho\}$ and $S_{2 x}(\rho)=\{z \in X /\|2 x-z\|=\rho\}$, with the continuous function $\varphi: K \rightarrow R$ defined by $\varphi(z)=$ $\|y+z\|-\|y+(2 x-z)\|$; we easily prove that $\varphi(2 x-z)=$ $-\varphi(z)$ so that the function $\varphi$ changes its sign on $K$ which is connected. Hence there is a vector $z_{0}$ with $\varphi\left(z_{0}\right)=0$, that is $\left\|y+z_{0}\right\|=\left\|y+\left(2 x-z_{0}\right)\right\|$, so the assertion is true if we put $z_{1}=z_{0}$ and $z_{2}=2 x-z_{0}$.

We remark that from this condition we deduce that for every nonnull vector, there exists a vector of equal norm which is also isosceles orthogonal to the given one; this is easily proved setting $x=0$ in condition (A).

Condition (B) is easily proved from Proposition 1 , using the auxiliary functional $\varphi(z)=\|y-z\|-\|y-(2 x-z)\|$. For $x=0$, condition (B) leads us to the same conclusion as in the case (A).

We remark that the pair of vectors $z_{1}, z_{2}$ and the pair of vectors $z_{3}, z_{4}$ in conditions (A) and (B) may be different from each other.

Condition $(\mathrm{C})$ requires that there exists a pair of vectors satisfying both conditions; this case is not satisfied in every normed space as we can see from the following counter example.

Example 2. Let $\left(R^{3},\|\cdot\|_{\infty}\right)$ be the normed space in which we define the norm

$$
\|x\|_{\infty}=\max \left(\left|x_{1}\right|,\left|x_{2}\right|,\left|x_{3}\right|\right) .
$$


In this space we prove that there exist $x, y \in X$ with $\|x\|<$ $\|y\|$ for which it is impossible to find vectors satisfying $(\mathrm{C})$. For this aim, let us consider $x=(-1 / 2,-1 / 2,-1 / 2)$ and $y=$ $(1,-1,0)$. Those vectors satisfy $\|x\|<\|y\|$ since $\|x\|=1 / 2$ and $\|y\|=1$. We use now condition $\left(\mathrm{C}^{\prime}\right)$.

The idea of the proof that there are not vectors satisfying condition $\left(\mathrm{C}^{\prime}\right)$ is as follows: we suppose by an absurd that there exists a vector $z=(\alpha, \beta, \gamma)$ with $\|y\|=\|z\|=\|2 x-z\|$ satisfying condition $\left(\mathrm{C}^{\prime}\right)$. We compute $2 x-z=(-1-\alpha,-1-$ $\beta,-1-\gamma)$; since $\|y\|=\|z\|=\|2 x-z\|=1$, we are forced to consider only particular values for $\alpha, \beta, \gamma$. In fact from

$$
\max (|\alpha|,|\beta|,|\gamma|)=\max (|-1-\alpha|,|-1-\beta|,|-1-\gamma|)=1,
$$

we remark that conditions: $-1 \leq \alpha \leq 0,-1 \leq \beta \leq 0,-1 \leq \gamma \leq$ 0 must be satisfied in a suitable manner.

First suppose that $\alpha=-1$; hence $\beta=0$ or $\gamma=0$ in order to satisfy (6). The following three cases hold: $z=(-1,0,0)$, $z=(-1,0, \gamma)$, and $z=(-1, \beta, 0)$, with $-1 \leq \beta \leq 0$ and $-1 \leq$ $\gamma \leq 0$.

In the first case compute $\|y+z\|=\max (0,-1,0)=1$ and $\|y+(2 x-z)\|=\max (1,-2,-1)=2$, so that this case is impossible.

In the second case compute $\|y+z\|=\max (0,-1, \gamma)=1$ and $\|y+(2 x-z)\|=\max (1,-2,-1-\gamma)=2$, so also this case is impossible.

In the third case compute $\|y+z\|=\max (0,-1+\beta, 0)=$ $|-1+\beta|$ and $\|y+(2 x-z)\|=\max (1,-2-\beta,-1)=|-2-\beta|$. We have $|-1+\beta|=|-2-\beta|$ only if $\beta=-1 / 2$, so that we have the vector $z=(-1,-1 / 2,0)$. We compute in this case $\|y+z\|=3 / 2$ and $\|y+(2 x-z)\|=3 / 2$, but $\|y-z\|=2$ and $\|y-(2 x-z)\|=1$; hence this vector does not satisfy the two conditions required.

All the remaining cases are similar, so we prove that in the space $\left(R^{3},\|\cdot\|_{\infty}\right)$, there exist no vectors satisfying condition (C).

Since the normed space of the previous example is a noninner product space, we purpose to find if condition (C) characterizes or not the inner product spaces among normed spaces. First we prove the sufficient condition.

Theorem 3. If $X$ is an inner product space, then $(C)$ is satisfied.

In fact, using the definition of the norm in inner product spaces and the basic property of the inner product this theorem may be easily proved.

The necessary condition remains an open problem, but in Section 5 we describe an interesting partial result.

\section{The Conditional Quadratic Functional Equation}

We give now a characterization of the quadratic equation on the restricted domain of equal-norm vectors in a normed space introduced in the previous section, that is a space in which condition $(\mathrm{C})$ is fulfilled.
Theorem 4. A function $f:(X,\|\cdot\|,(C)) \rightarrow R$ is a solution of the conditional equation (3) if and only if $f$ is everywhere quadratic, that is, (1).

Proof. It is easily seen that (1) implies (3).

Suppose now that (3) is satisfied. First we prove the following relations:

$$
\begin{gathered}
f(0)=0, \\
f(2 x)=4 f(x), \\
f(x)=f(-x) .
\end{gathered}
$$

It is sufficient to put $x=y=0, x=y$ and choose the pair $x,-y$ in (3), respectively.

Let $x, y \in X$ be arbitrarily given vectors with $\|x\|<\|y\|$. For property (C), there exist the vectors $x_{1}, x_{2} \in X$ such that $2 x=x_{1}+x_{2}$ and

$$
\begin{gathered}
\|y\|=\left\|x_{1}\right\|=\left\|x_{2}\right\|, \\
\left\|y+x_{1}\right\|=\left\|y+x_{2}\right\|, \\
\left\|y-x_{1}\right\|=\left\|y-x_{2}\right\| .
\end{gathered}
$$

Substituting in (3) $x_{1}, x_{2}$ in place of $x, y$, we obtain

$$
\begin{aligned}
& f\left(x_{1}+x_{2}\right)+f\left(x_{1}-x_{2}\right)=2 f\left(x_{1}\right)+2 f\left(x_{2}\right), \text { that is, } \\
& f\left(x_{1}-x_{2}\right)=2 f\left(x_{1}\right)+2 f\left(x_{2}\right)-f(2 x) .
\end{aligned}
$$

Since $f$ is even, for (3), (8), and (11), we get

$$
\begin{aligned}
f(2 x+ & 2 y)+f(2 x-2 y) \\
= & f\left[\left(y+x_{1}\right)+\left(y+x_{2}\right)\right]+f\left[\left(y-x_{1}\right)+\left(y-x_{2}\right)\right] \\
= & 2 f\left(y+x_{1}\right)+2 f\left(y+x_{2}\right)-f\left(y+x_{1}-y-x_{2}\right) \\
& +2 f\left(y-x_{1}\right)+2 f\left(y-x_{2}\right)-f\left(y-x_{1}-y+x_{2}\right) \\
= & 2 f\left(y+x_{1}\right)+2 f\left(y-x_{1}\right)+2 f\left(y+x_{2}\right) \\
& +2 f\left(y-x_{2}\right)-2 f\left(x_{1}-x_{2}\right) \\
= & 8 f(y)+4 f\left(x_{1}\right)+4 f\left(x_{2}\right) \\
& -4 f\left(x_{1}\right)-4 f\left(x_{2}\right)+2 f(2 x) \\
= & 2 f(2 x)+2 f(2 y) .
\end{aligned}
$$

The proof is similar in case of $\|x\|>\|y\|$ : it is sufficient to change the role of $x$ and $y$, since $f$ is even.

\section{The D'Alembert Conditional Functional Equation}

We give now a characterization of the D'Alembert equation on the restricted domain of equal-norm vectors in a normed space introduced in the previous section, that is, a space in which condition (C) is fulfilled. 
Theorem 5. A function $f:(X,\|\cdot\|,(C)) \rightarrow R$ is a solution of the conditional equation (4) if and only if $f$ satisfies everywhere the D'Alembert equation, that is, (2).

Proof. It is easily seen that (2) implies (4).

Suppose now that (4) is satisfied. First we prove that $f(0)=0$ or $f(0)=1$, putting in (4) $x=y=0$ and obtaining $f^{2}(0)=f(0)$.

(a) Let $f(0)=0$; we shall prove that, in this case, $f(x)=0$ for all $x \in X$. Setting in (4) $x=y$, we get

$$
f(2 x)=2 f^{2}(x) .
$$

Then, substituting in (4) $2 x$ and $2 y$ in place of $x, y$, we get $f(2(x+y))+f(2(x-y))=2 f(2 x) f(2 y)$ for $\|x\|=\|y\|$, and using (13) we have

$$
\|x\|=\|y\| \Longrightarrow f^{2}(x+y)+f^{2}(x-y)=4 f^{2}(x) f^{2}(y) .
$$

On the other hand, squaring both sides of (4), we get

$$
\begin{aligned}
\|x\|=\|y\| \Longrightarrow & f^{2}(x+y)+f^{2}(x-y) \\
& +2 f(x+y) f(x-y)=4 f^{2}(x) f^{2}(y) .
\end{aligned}
$$

Comparing (14) with (15), we have

$$
\|x\|=\|y\| \Longrightarrow f(x+y) f(x-y)=0 .
$$

Now, let $x \in X$ be an arbitrary vector. Then there exists a vector $y_{0} \in X$ such that $\|x\|=\left\|y_{0}\right\|$ and $\left\|x+y_{0}\right\|=\left\|x-y_{0}\right\|$ (see Szabó, [6, Page 270]). Substituting $x+y_{0}$ and $x-y_{0}$ for $x$ and $y$, respectively, in (4), we get $f(2 x)+f\left(2 y_{0}\right)=2 f(x+$ $\left.y_{0}\right) f\left(x-y_{0}\right)$, and then by (16), $f(2 x)+f\left(2 y_{0}\right)=0$. Hence, by (13), we get $f^{2}(x)+f^{2}\left(y_{0}\right)=0$; that is, $f(x)=0$.

(b) Let $f(0)=1$, we put $x=y$ in (4) and obtain

$$
f(2 x)=2 f^{2}(x)-1 \text {. }
$$

We now prove that $f$ is even. Substitution in (4) of $x / 2$ and $-x / 2$ for $x$ and $y$ leads to $1+f(x)=2 f(x / 2) f(-x / 2)$, for all $x \in X$. Then, putting $x=-x$ in this last equation, we get $1+f(-x)=2 f(-x / 2) f(x / 2)$; hence $f(x)=f(-x)$.

Using (17) and (4), we prove the following useful equations:

$$
\begin{gathered}
\|x\|=\|y\| \Longrightarrow f(x+y) f(x-y)=f^{2}(x)+f^{2}(y)-1, \\
\|x\|=\|y\| \Longrightarrow f(x)+f(y)=2 f\left(\frac{x+y}{2}\right) f\left(\frac{x-y}{2}\right) .
\end{gathered}
$$

Proof of (18). Substituting in (4) $2 x$ and $2 y$ in place of $x, y$, we get $f(2(x+y))+f(2(x-y))=2 f(2 x) f(2 y)$ for $\|x\|=\|y\|$, and using (17) we have

$$
\begin{aligned}
& \|x\|=\|y\| \\
& \Longrightarrow f^{2}(x+y)+f^{2}(x-y)-1 \\
& \quad=4 f^{2}(x) f^{2}(y)-2 f^{2}(x)-2 f^{2}(y)+1 .
\end{aligned}
$$

On the other hand, squaring both sides of (4), we get (15). Then, comparing (20) and (15) we prove (18).

Proof of (19). Substituting in (18) $x / 2$ and $y / 2$ in place of $x, y$, we get $f((x+y) / 2) f((x-y) / 2)=f^{2}(x / 2)+f^{2}(y / 2)-1$; that is, $2 f((x+y) / 2) f((x-y) / 2)=2 f^{2}(x / 2)-1+2 f^{2}(y / 2)-1$, and using (17) in the right hand side, we have (19).

Now we have to prove that $f$ satisfies the D'Alembert functional equation for all $x, y \in X$.

Let $x, y \in X$ be arbitrarily given vectors; we may assume that $\|x\|<\|y\|$. We distinguish two cases: first $\|x+y\|=\|x-y\|$ and then $\|x+y\| \neq\|x-y\|$.

(a) $\|x+y\|=\|x-y\|$. Using (4) with $x+y$ and $x-y$ in place of $x$ and $y$, respectively, we have $f(2 x)+f(2 y)=$ $2 f(x+y) f(x-y)$, and then by (17) we get

$$
f^{2}(x)+f^{2}(y)=f(x+y) f(x-y)+1 .
$$

Moreover, since $\|(x+y) / 2\|=\|(x-y) / 2\|$, substituting $(x+$ $y) / 2$ and $(x-y) / 2$ in place of $x$ and $y$, respectively, in (4) we obtain $f(x)+f(y)=2 f((x+y) / 2) f((x-y) / 2)$. Squaring both sides of this last equation and using (21) we get

$$
\begin{gathered}
f(x+y) f(x-y)+2 f(x) f(y)+1 \\
=4 f^{2}\left(\frac{x+y}{2}\right) f^{2}\left(\frac{x-y}{2}\right) .
\end{gathered}
$$

From (17) we have $f(x \pm y)=2 f^{2}((x \pm y) / 2)-1$, so that substitution of $f^{2}((x \pm y) / 2)$ in the right hand side of (22) leads to $f(x+y) f(x-y)+2 f(x) f(y)+1=[f(x+y)+$ $1][f(x-y)+1]$, which is the D'Alembert functional equation for this values of $x, y$.

(b) Let us now suppose that $\|x+y\| \neq\|x-y\|$ and use the auxiliary vector $z$ of condition $\left(\mathrm{C}^{\prime}\right)$.

Since $\|y\|=\|z\|$, from (4) we get

$$
f(y+z)+f(y-z)=2 f(y) f(z)
$$

and then by $\|y\|=\|2 x-z\|$ we obtain in the same way

$$
f(y+2 x-z)+f(y-(2 x-z))=2 f(y) f(2 x-z) .
$$

Addition of both sides of (23) and (24) leads to

$$
\begin{aligned}
& f(y+z)+f(y-z)+f(y+2 x-z)+f(y-(2 x-z)) \\
& \quad=2 f(y)[f(z)+f(2 x-z)] .
\end{aligned}
$$

Now, writing in (19) $z$ and $2 x-z$ in place of $x$ and $y$, respectively, since $\|z\|=\|2 x-z\|$, and from the evenness of $f$, we arrive at

$$
f(z)+f(2 x-z)=2 f(x) f(x-z) .
$$

In the same way, by $\|y+z\|=\|y+(2 x-z)\|$ and $\|y-z\|=$ $\|y-(2 x-z)\|$, we obtain from (19)

$$
\begin{aligned}
& f(y+z)+f(y+(2 x-z))=2 f(x+y) f(x-z), \\
& f(y-z)+f(y-(2 x-z))=2 f(x-y) f(x-z) .
\end{aligned}
$$


Adding both sides of (27) and then using (25) and (26), we get

$$
[f(x+y)+f(x-y)] f(x-z)=2 f(x) f(y) f(x-z) .
$$

We have to deal with the following possibilities: $f(x-z) \neq 0$ or $f(x-z)=0$.

In the first case, that is, $f(x-z) \neq 0$, we prove that the D'Alembert functional equation is satisfied for these values of $x, y \in X$.

In the second case we suppose that $f(x-z)=0$. From (17) we deduce that $f((x-z) / 2) \neq 0$ and $f((x-z) / 4) \neq 0$ by a simple calculation. Hence, we can easily show that (28) can be reformulated as follows:

$$
\begin{gathered}
{\left[f\left(\frac{x+y}{2}\right)+f\left(\frac{x-y}{2}\right)\right] f\left(\frac{x-z}{2}\right)} \\
=2 f\left(\frac{x}{2}\right) f\left(\frac{y}{2}\right) f\left(\frac{x-z}{2}\right) \\
{\left[f\left(\frac{x+y}{4}\right)+f\left(\frac{x-y}{4}\right)\right] f\left(\frac{x-z}{4}\right)} \\
=2 f\left(\frac{x}{4}\right) f\left(\frac{y}{4}\right) f\left(\frac{x-z}{4}\right)
\end{gathered}
$$

so that we obtain

$$
\begin{gathered}
f\left(\frac{x+y}{2}\right)+f\left(\frac{x-y}{2}\right)=2 f\left(\frac{x}{2}\right) f\left(\frac{y}{2}\right), \\
f\left(\frac{x+y}{4}\right)+f\left(\frac{x-y}{4}\right)=2 f\left(\frac{x}{4}\right) f\left(\frac{y}{4}\right) .
\end{gathered}
$$

Squaring both side of (30) and we get

$$
\begin{gathered}
f^{2}\left(\frac{x+y}{2}\right)+f^{2}\left(\frac{x-y}{2}\right)+2 f\left(\frac{x+y}{2}\right) f\left(\frac{x-y}{2}\right) \\
=4 f^{2}\left(\frac{x}{2}\right) f^{2}\left(\frac{y}{2}\right)
\end{gathered}
$$

and by elementary calculations

$$
\begin{aligned}
2 f^{2} & \left(\frac{x+y}{2}\right)-1+2 f^{2}\left(\frac{x-y}{2}\right)-1 \\
& +4 f\left(\frac{x+y}{2}\right) f\left(\frac{x-y}{2}\right) \\
& =8 f^{2}\left(\frac{x}{2}\right) f^{2}\left(\frac{y}{2}\right)-2
\end{aligned}
$$

in order to obtain, using (17),

$$
\begin{gathered}
f(x+y)+f(x-y)+4 f\left(\frac{x+y}{2}\right) f\left(\frac{x-y}{2}\right) \\
=2\left[4 f^{2}\left(\frac{x}{2}\right) f^{2}\left(\frac{y}{2}\right)-1\right] .
\end{gathered}
$$

Now let us consider the following identity:

$$
\begin{aligned}
4 f^{2}\left(\frac{x}{2}\right) f^{2}\left(\frac{y}{2}\right)-1= & \left\{2 f^{2}\left(\frac{x}{2}\right)-1\right\}\left\{2 f^{2}\left(\frac{y}{2}\right)-1\right\} \\
& +2 f^{2}\left(\frac{x}{2}\right)-1+2 f^{2}\left(\frac{y}{2}\right)-1
\end{aligned}
$$

by (17) we get $4 f^{2}(x / 2) f^{2}(y / 2)-1=f(x) f(y)+f(x)+f(y)$. We substitute the right hand side of this last equation in the right hand side of (34) and have

$$
\begin{gathered}
f(x+y)+f(x-y)+4 f\left(\frac{x+y}{2}\right) f\left(\frac{x-y}{2}\right) \\
=2 f(x) f(y)+2 f(x)+2 f(y) .
\end{gathered}
$$

We purpose now to prove the following identity:

$$
2 f\left(\frac{x+y}{2}\right) f\left(\frac{x-y}{2}\right)=f(x)+f(y)
$$

in order to show that the D'Alembert functional equation is satisfied in this case too.

Squaring both sides of (31) we have $f^{2}((x+y) / 4)+f^{2}((x-$ $y) / 4)+2 f((x+y) / 4) f((x-y) / 4)=4 f^{2}(x / 4) f^{2}(y / 4)$, and by the suitable calculation above used, we get $\left[2 f^{2}((x+y) / 4)-\right.$ $1]+\left[2 f^{2}((x-y) / 4)-1\right]+4 f((x+y) / 4) f((x-y) / 4)=$ $2\left[2 f^{2}(x / 4)-1\right]\left[2 f^{2}(y / 4)-1\right]-2\left[2 f^{2}(x / 4)-1\right]-2\left[2 f^{2}(y / 4)-\right.$ $1]$.

Now let us use (17) in order to have $f((x+y) / 2)+f((x-$ $y) / 2)+4 f((x+y) / 4) f((x-y) / 4)=2 f(x / 2) f(y / 2)+$ $2 f(x / 2)+2 f(y / 2)$.

We now consider (30) and we can prove that $2 f((x+$ $y) / 4) f((x-y) / 4)=f(x / 2)+f(y / 2)$, by the same techniques used in the proof of (37). Squaring both sides of this last equation we have $4 f^{2}((x+y) / 4) f^{2}((x-y) / 4)=f^{2}(x / 2)+$ $f^{2}(y / 2)+2 f(x / 2) f(y / 2)$ and by suitable calculations using (17) and (30) we prove (37).

Hence the theorem is proved.

\section{The Isosceles Orthogonal Additive Functional Equation}

In order to study the problem of the characterization of the normed spaces satisfying condition $(\mathrm{C})$, we recall the following result of Szabó [8] concerning a characterization of the inner product spaces among the normed spaces; its characterization involves a particular conditional functional equation.

Theorem 6 (Szabó). There exists a nontrivial, even, isosceles orthogonally additive mapping from $X$ to $Y$ if and only if $X$ is an inner product space.

Until now we can prove a partial result: we describe two properties of the isosceles orthogonally additive mappings in the normed spaces $(X,\|\cdot\|,(C))$.

Theorem 7. Let $f:(X,\|\cdot\|,(C)) \rightarrow R$ be an even isosceles orthogonally additive mapping, that is, an even solution of the conditional equation

$$
\|x+y\|=\|x-y\| \Longrightarrow f(x+y)=f(x)+f(y),
$$

then $f$ satisfies everywhere the quadratic equation, that is, (1), and it is constant on each sphere centred at zero.

Proof. Let $f$ be an even isosceles orthogonally additive mapping. From $x \perp_{i} y$, we may show that $x \perp_{i}(-y)$; hence 
from (38), in which we substitute $x$ and $-y$ in place of $x$ and $y$, we get $f(x-y)=f(x)+f(y)$, using the oddness of $f$. Adding both sides of (38) and this last equation, we have

$$
\begin{aligned}
& \|x+y\|=\|x-y\| \\
& \Longrightarrow f(x+y)+f(x-y)=2 f(x)+2 f(y) .
\end{aligned}
$$

Now, consider an arbitrary $x \in X$. We prove that for all $x \in X$

$$
f(2 x)=4 f(x) .
$$

We observe that for all $x \in X$, there exists $z \in X$ such that $x \perp_{i}( \pm z)$ and $x+z \perp_{i} \pm(x-z)$; it is sufficient to consider the continuous functional $\varphi: S(\|x\|) \rightarrow R$ defined by $\varphi(u)=$ $\|x+u\|-\|x-u\|$. This functional has a connected domain and satisfies $\varphi(-u)=-\varphi(u)$; hence there exists a vector $z \in S(\|x\|)$ such that $\varphi(z)=0$.

From (38), using the evenness of the function $f$, we get

$$
\begin{aligned}
f(2 x) & =f(x+z+x-z) \\
& =f(x+z)+f(x-z) \\
& =2 f(x)+2 f(z) .
\end{aligned}
$$

On the other hand, since $((x+z) / 2) \perp_{i} \pm(x-z) / 2$, from (38) and the evenness of $f$ again, we have

$$
\begin{aligned}
f(x) & =f\left(\frac{x+z}{2}+\frac{x-z}{2}\right)=f\left(\frac{x+z}{2}\right)+f\left(\frac{x-z}{2}\right) \\
& =f\left(\frac{x+z}{2}\right)+f\left(\frac{z-x}{2}\right)=f\left(\frac{x+z+z-x}{2}\right) \\
& =f(z) .
\end{aligned}
$$

Thus, substituting $f(x)$ in place of $f(z)$ in (41) we obtain (40).

Let us now consider $x, y \in(X,\|\cdot\|,(C))$ with $\|x\|=\|y\|$. Putting

$$
\begin{aligned}
& u=x+y, \\
& v=x-y
\end{aligned}
$$

we have $x=(u+v) / 2, y=(u-v) / 2$, that is, $u \perp_{i} v$. From (39) we obtain $f(u+v)+f(u-v)=2 f(u)+2 f(v)$; hence, $f(2 x)+f(2 y)=2 f(x+y)+2 f(x-y)$. From (40) we deduce that (3) is true in this case. So Theorem 4 gives us that $f$ is everywhere quadratic.

Let us now prove that $f$ is constant on each sphere centred at the origin. Let $x, y \in(X,\|\cdot\|,(C))$ be such that $\|x\|=\|y\|$. By (43), substituting $u, v$ in place of $x, y$ in (38), we have $f(u+$ $v)=f(u)+f(v)$; hence $f(2 x)=f(x+y)+f(x-y)$. We now remember that $f$ satisfies the quadratic equation everywhere, in order to show that $f(2 x)=2 f(x)+2 f(y)$; then, for (40) we obtain that $f(x)=f(y)$ for all $x, y \in(X,\|\cdot\|,(C))$ with $\|x\|=\|y\|$. Consequently, there exists a function $\varphi: R \rightarrow R$ such that $f(x)=\phi(\|x\|)$ for all $x \in(X,\|\cdot\|,(C))$. The theorem is proved.

\section{References}

[1] C. Alsina and J. L. Garcia Roig, "On a conditional cauchy equation on rhombuses," in Functional Analysis, Approximation Theory and Numerical Analysis, pp. 5-7, World Scientific Publishing Company, River Edge, NJ, USA, 1994.

[2] S. Gudder and D. Strawther, "Orthogonally additive and orthogonally increasing functions on vector spaces," Pacific Journal of Mathematics, vol. 58, no. 2, pp. 427-436, 1975.

[3] J. Rätz, "On orthogonally additive mappings," Aequationes Mathematicae, vol. 28, no. 1-2, pp. 35-49, 1985.

[4] Gy. Szabó, “ $\phi$-orthogonally additive mappings I," Acta Mathematica Hungarica, vol. 58, no. 1-2, pp. 101-111, 1991.

[5] L. Paganoni and J. Rätz, "Conditional functional equations and orthogonal additivity," Aequationes Mathematicae, vol. 50, no. 1-2, pp. 135-142, 1995.

[6] Gy. Szabó, "A conditional cauchy equation on normed spaces," Publicationes Mathematicae Debrecen, vol. 42, no. 3-4, pp. 265271, 1993.

[7] R. C. James, “Orthogonality in normed linear spaces," Duke Mathematical Journal, vol. 12, pp. 291-302, 1945.

[8] Gy. Szabó, "Isosceles orthogonally additive mappings and inner product spaces," Publicationes Mathematicae Debrecen, vol. 46, no. 3-4, pp. 373-384, 1995. 


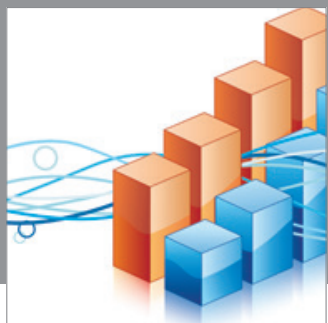

Advances in

Operations Research

mansans

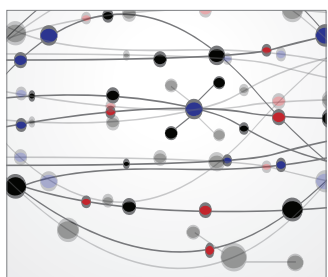

The Scientific World Journal
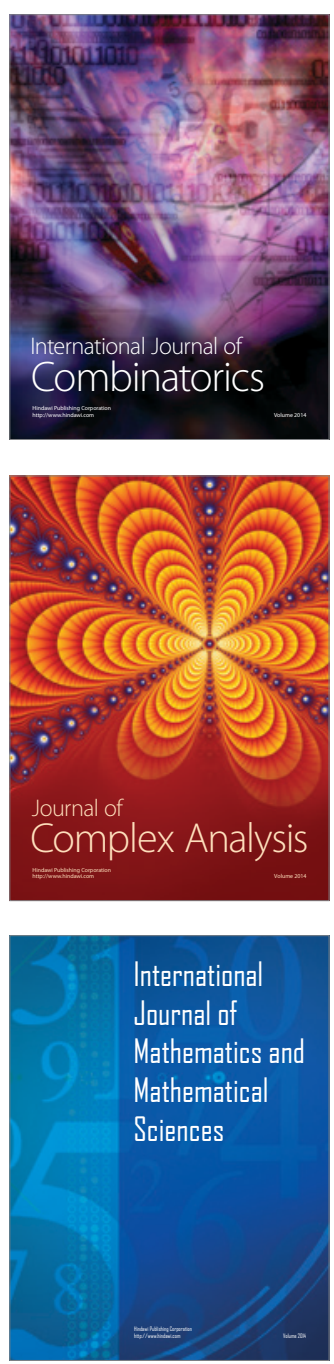
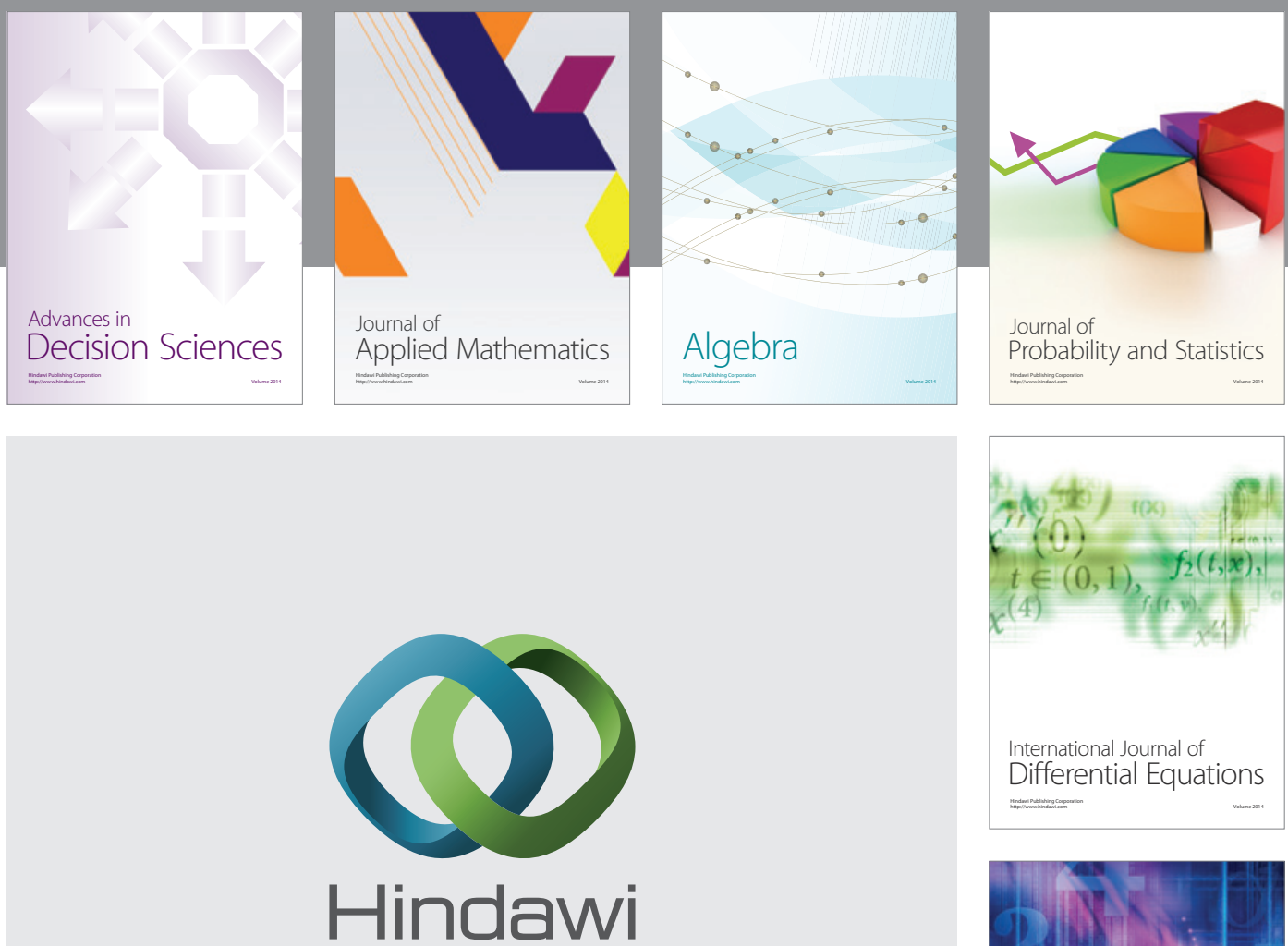

Submit your manuscripts at http://www.hindawi.com
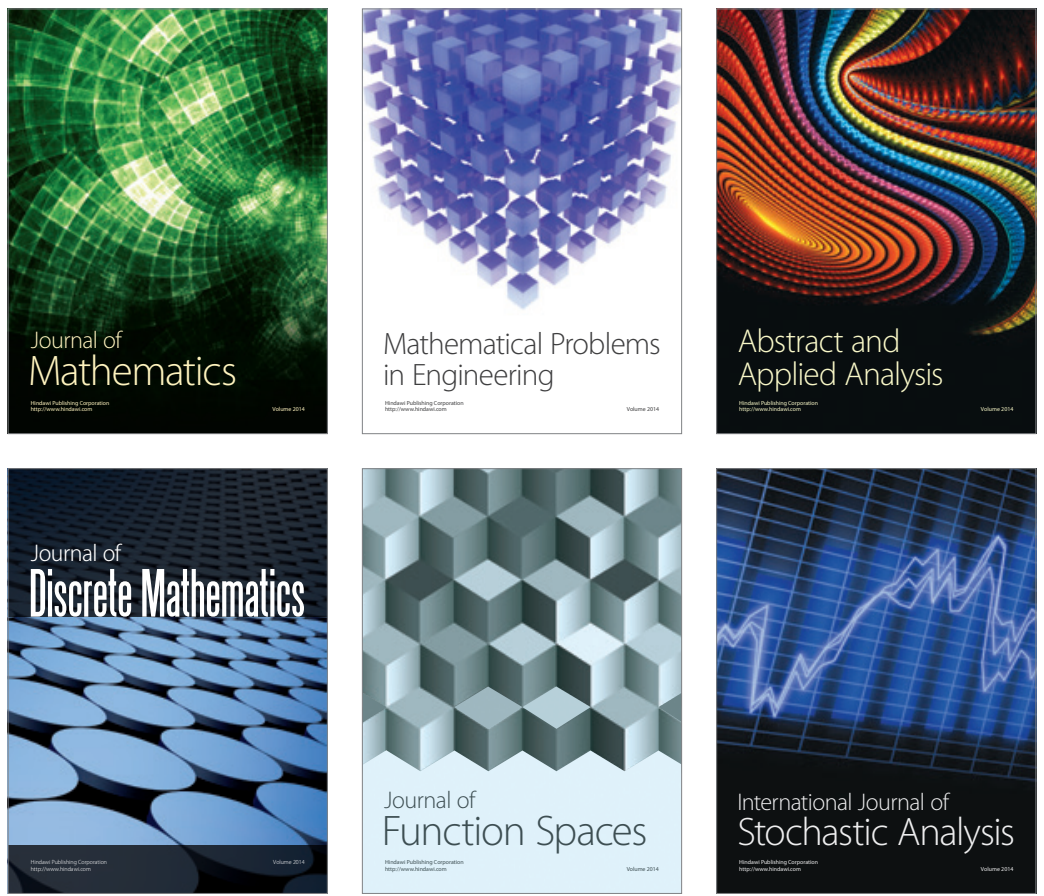

Journal of

Function Spaces

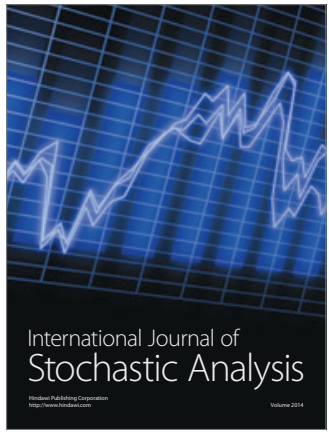

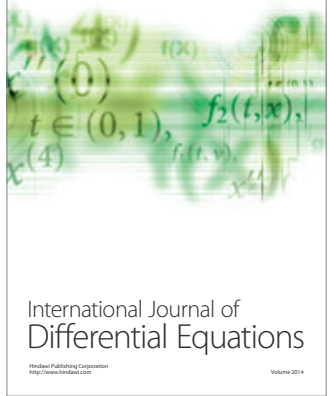
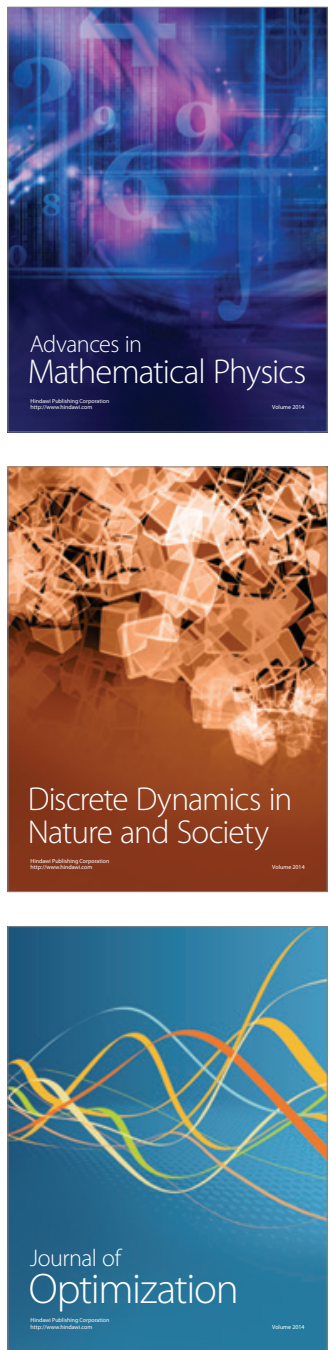